\title{
Fluor vaginalis
}

Unspezifischer Fluor vaginalis und milde, nicht rezidivierende Vaginosen sind Indikationen für eine Behandlung mit Naturheilverfahren. Begleitend können Naturheilverfahren auch bei einer pathogenen rezidivierenden Besiedlung eingesetzt werden.

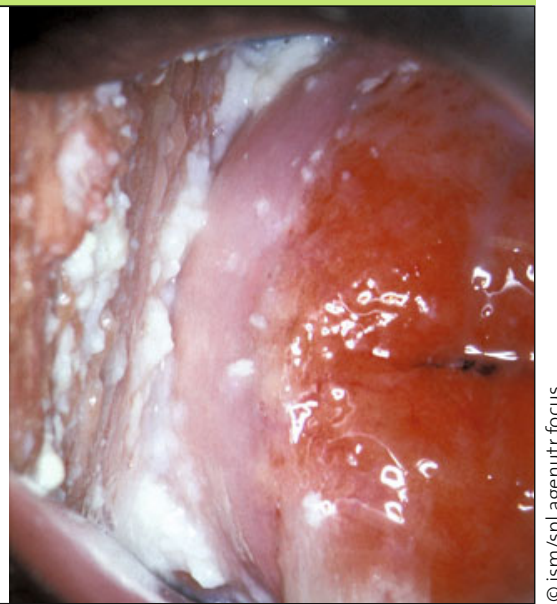

\section{Hydro-Thermo-Therapie}

- Sitzbäder (Salhumin Sitzbad N) können mit $30-35^{\circ} \mathrm{C}$ ein- bis zweimal tgl. durchgeführt werden.

-Zugesetzte Kamille wirkt kühlend und juckreizlindernd (Kamillosan Konzentrat Lösung $15 \mathrm{ml} / \mathrm{l}$ Wasser bei einem Halbbad). Cave: Allergische Reaktionen!

- Bei rezidivierenden Infekten sind Vaginalduschen, welche in der Apotheke erhältlich sind (Multi Gyn. Vag. Duschen) mit Leitungswasser oder Zusätzen (Multi Gyn. Vag. Brausetbl.) einfach anwendbar.

Übertriebene Waschhandlungen verschlimmern die Symptome.

Bei Stress (Prüfungen bei Studentinnen, berufliche Belastungen, Überforderung im Haushalt, Beziehungsprobleme etc.) unterstützt Bewegungstherapie die körperliche Aktivierung und erleichtert die Fluorbehandlung.

\section{Ernährungstherapie}

Im Rahmen der Ernährungstherapie steht zunächst die mikrobiologische Therapie („Darmsanierung") im Vordergrund, da eine pathogene Darmbesiedlung Vaginalinfektionen verursachen kann. Eine Darmmykose muss stets mitbehandelt werden, da sich sonst die Vaginalmykose nicht dauerhaft heilen lässt (z.B. Mutaflor).

Eine mediterrane Diät mit regelmäßigen Fischmahlzeiten sowie die Verwendung von hochwertigen Ölen ver- mindern aufgrund des Gehalts an Omega-3-Fettsäuren entzündliche Reaktionen. Begrenzung tierischer Lebensmittel reduziert die Zufuhr von Arachidonsäure, die Ausgangspunkt für proinflammatorisch wirkende Eicosanoide ist.

\section{Phythotherapie}

Phytotherapeutisch hat sich die täglicheTrinkmenge von drei bis vier Tassen Lapacho-Tee, der nachweislich fungizid wirkt, bewährt.

Für die lokale Behandlung hat die Taubnessel beim unspezifischen Fluor albus eine positive Monografie der Kommission E erhalten. Bei verstärktem Ausfluss ohne weitere Krankheits-

\section{Man nehme}

\section{Rezept für Tee- und Sitzbadmischung}

Weiße Taubnesselblüten Frauenmantelkraut Schafgarbenkraut $60,0 \mathrm{~g}$ Zinnkraut $40,0 \mathrm{~g}$

2 EL der Teemischung mit $1000 \mathrm{ml}$ kochendem Wasser überbrühen und $15 \mathrm{~min}$. ziehen lassen, durch ein Teesieb gießen. Mehrmals tgl. eine Tasse trinken oder als Sitzbad anwenden. Ein paar Tropfen von reinem, ätherischen Teebaumöl dem Sitzbad zugegeben verstärkt die Wirkung. symptome ist oft nur das Scheidenmilieu leicht gestört. Hier helfen Vaginalzäpfchen mit Milchsäurebakterien oder mit Majoranzusatz, die die natürliche Vaginalflora wieder herstellen (Symbio Vag. Vaginalzäpfchen; Majorana/Melissa Vag. Tbl.), oder mit Rosenöl getränkte Tampons.

\section{Ordnungstherapie}

Im Rahmen der Ordnungstherapie sind bei häufigen Infekten folgende Aspekte mit der Patientin zu besprechen:

(1) Das Tragen von Slipeinlagen, möglichst ohne Kunststofffolie und Duftstoffe, ist angezeigt.

2Unterwäsche aus Baumwolle muss durch zehnminütiges Kochen desinfiziert werden.

(3) Durch häufiges Rasieren, Intim-Piercing und das Tragen von String-Tangas können sich die Symptome verstärken. 4 Bei der Pflege der Haut gilt: „Waschen, Trocknen, Cremen“. Wir empfehlen Melkfett mit Zusatz Ringelblumensalbe. Auch Rosenöl kann aufgrund seiner Allergenarmut für den äußeren Intimbereich verwendet werden.

(5) Als Schutz vor Vaginalinfekten und bei Schwangeren haben sich vor Saunaoder Schwimmbadbesuchen auch ellen ${ }^{\circledR}$ Probiotic Tampons ${ }^{\circledR}$ oder das Bade- und Schutztampon Symbiofem Protect bewährt.

- Dr. med. Beatrice Nobis, Aachen 